\title{
GW23-e0957 CLINICAL APPLICATION OF EMERGENCY BEDSIDE TEMPORARY CARDIAC PACING WITHOUT X-RAY FLUOROSCOPY
}

doi:10.1136/heartjnl-2012-302920n.12

Wei Xiao-jun, Liao Wei, Zhong Yi-ming, Wei Xiao-jun. Department of Cardiology,

Affiliated Hospital of Gannan Medical College, GanZhou, JiangXi 341000

Objectives To investigate the clinical value and safety of emergency bedside temporary cardiac pacing without X-ray fluoroscopy.

Methods 63 patients were selected due to ventricular rate less than or equal to 40 times/min and (or) RR interval greater than $3.0 \mathrm{~s}$ caused by various diseases and associated with severe hemodynamic disorders, Adams syndrome attack or disturbance of consciousness. Subclavian vein was punctured for vasodilating sheath placement by seldinger technique without $\mathrm{x}$-ray fluoroscopy at bedside. Temporary pacing electrode was inserted into the right ventricular cavity from the sheath and success was judged by three methods based on intracardiac ECG, pacing ECG, or whether it could induce PVCs.

Results Out of 63 patients, 59 cases of pacing was successful and the success rate was $93.7 \%$. The operation time from the puncture to the successful pacing was about 2-15 $\mathrm{min}$ (average $4.5 \mathrm{~min}$ ) and pacing thresholds were less than or equal to $2.0 \mathrm{~V}$. The hemodynamic significantly improved, Adams syndrome attacks stopped and consciousness returned to normal in successful pacing patients. No serious complications occurred such as infective endocarditis, bleeding, pneumothorax, hemothorax and so on.

Conclusions Emergency bedside temporary cardiac pacing without x-ray fluoroscopy is important rescue of severe bradycardia or cardiac arrest, particularly suitable for critically ill patients, who should not be moved and need emergency pacing. It has fast, easy and safe advantages and is indispensable means in the cardiac emergency work. 